\title{
ROL DE LA SUPERFAMILIA DEL FACTOR DE CRECIMIENTO TRANSFORMANTE B EN EL OVARIO Y SU RELACIÓN CON LA PATOGENIA DE LA ENFERMEDAD QUÍSTICA OVÁRICA BOVINA
}

\author{
Matiller, V. ${ }^{1,2,3}$; DiaZ, P. U. ${ }^{1,4}$; StangaferRo, M. L. ${ }^{1,5}$; \\ Rodriguez, F. M. ${ }^{1,6}$; Ortega, H. H. ${ }^{1,6} ;$ ReY, . $^{1,6}$ \& Salvetti, N. R. ${ }^{1,6}$
}

\begin{abstract}
RESUMEN
En los últimos años se ha avanzado en la búsqueda por esclarecer los complejos mecanismos de control intraováricos que, concomitantemente con señales sistémicas, coordinan el reclutamiento, la selección y el crecimiento de los folículos desde el estadio primordial hasta la ovulación y formación del cuerpo lúteo. Una gran cantidad de factores de crecimiento, muchos de ellos pertenecientes a la Superfamilia del Factor de Crecimiento Transformante Beta (TGFB) son expresados por las células somáticas ováricas y ovocitos en desarrollo, ejecutando una función de regulación intraovárica en la foliculogénesis. La alteración de estos factores podría contribuir a la patogenia de enfermedades reproductivas como la enfermedad quística ovárica (EQO), uno de los desórdenes reproductivos más frecuentes en el ganado bovino lechero. Esta enfermedad provoca cuantiosas pérdidas para la producción pecuaria. Los avances en la comprensión de los mecanismos de regulación intraováricos facilitan el desarrollo de nuevos enfoques para el control y la manipulación de la función ovárica y la mejora de la fertilidad en el ganado doméstico.

Palabras clave: Superfamilia de TGFB, foliculostatina, ovario, bovinos, enfermedad quística ovárica.
\end{abstract}

\footnotetext{
1.- Laboratorio de Biología Celular y Molecular Aplicada. ICIVET Litoral (UNL-CONICET). Kreder 2805. (3080) Esperanza, provincia de Santa Fe.

2.- Cátedra de Base de Nutrición y Alimentación Animal. Facultad de Ciencias Veterinarias (UNL). Kreder 2805. (3080) Esperanza, provincia de Santa Fe.

3.- Cátedra de Nutrición de Monogástricos. FCV (UNL)

4.- Cátedra de Farmacología. FCV (UNL)

5.- Cátedra de Teriogenología. FCV (UNL)

6.- Cátedra de Biología Celular. FCV (UNL)

Manuscrito recibido el 27 de noviembre de 2013 y aceptado para su publicación el $1^{\circ}$ de abril de 2014 .
} 


\section{SUMMARY}

\section{Role of transforming growth factor B superfamily in ovarian and its relation to the pathogenesis of bovine cystic ovarian disease.}

In recent years, progress has been made in the quest to elucidate the complex mechanisms of intraovarian control which, concomitantly with systemic signs, coordinate the recruitment, selection and growth of follicles from the primordial stage until ovulation, and corpus luteum formation. A large number of growth factors, many of them belonging to the Superfamily Transforming Growth Factor Beta (TGFB), are expressed by ovarian somatic cells and oocytes in development, exerting an intra-ovarian regulating function in folliculogenesis. The alteration of these factors may contribute to the pathogenesis of reproductive diseases such as cystic ovarian disease (COD), one of the most common reproductive disorders in dairy cattle. This disease causes considerable losses to livestock production. Advances in understanding the mechanisms of intraovarian regulation facilitates the development of new approaches for the control and manipulation of ovarian function and fertility improvement in domestic livestock.

Key words: TGFB superfamily, follistatin, ovary, bovine, cystic ovarian disease.

\section{INTRODUCCIÓN}

Los factores de crecimiento son polipéptidos y proteínas semejantes a las hormonas, que actúan predominantemente en forma paracrina y autocrina, y participan en la promoción de la actividad mitogénica necesaria para la proliferación y remodelación del tejido local. Su mecanismo de acción consiste en provocar respuestas celulares mediante la unión a receptores específicos en la superficie celular en su tejido blanco (Hafez \& Hafez, 2004). Existen diversas familias de factores de crecimiento con estructuras bioquímicas similares. En cuanto a sus funciones, aunque muchos de estos estimulan la proliferación celular, unos pocos tienen funciones inhibidoras, tales como el factor de crecimiento transformante $\beta$ (TGFB). Estudios recientes han demostrado que los factores de crecimiento también regulan diversas proteínas en el ciclo celular, y pueden tener un efecto directo o indirecto sobre la proliferación celular. Los análisis moleculares de los factores de crecimiento, incluyendo la clonación y secuenciación de los receptores específicos han contribuido, en gran medida a dilucidar la función que estos factores juegan en la homeostasis celular y el desarrollo neoplásico (Lloyd, 1997).

La superfamilia del TGFB es una agrupación muy grande de proteínas multifuncionales que afectan diversos procesos celulares que van desde la regulación de la diferenciación y proliferación celular, hasta procesos fisiológicos complejos como la inflamación y la cicatrización de tejidos, incluyendo también a la formación del hueso, entre otros. Además, alteraciones en sus señales pueden contribuir a la patogénesis de enfermedades tan diversas como las autoinmunes, la fibrosis y el cáncer (Sosa Garrocho y Macias Silva et al., 2004). Varios miembros de esta superfamilia juegan un destacado papel en el desarrollo del sistema nervioso e incluso algunos están regulados por actividad neuronal. Por ejemplo, durante el desarrollo de la neocorteza en mamíferos,

8 | Revista FAVE - Ciencias Veterinarias 13 (1-2) 2014 